Historic, Archive Document

Do not assume content reflects current scientific knowledge, policies, or practices. 



\title{
Wholesale Price List of Peonies for September and October Planting.
}

\author{
We Issue No Catalogue.
}

Quotations are F. O. B. Ashtabula, Ohio. Cash with order. No charge for packing. We furnish three eye divisions, sometimes more. 5 roots at 10 rate and 25 roots at 100 rate, of one kind.

We prefer to ship in clumps and you divide as you wish and offer three good stems as equal to one divided root at prices named below.

Rating

8.7 Albatre, White

8.8 Alsace Lorraine, White, late

8.7 Bayadere, White

8.1 Couronne de Or, White, late

7.8 Duc de Wellington, White, late

8.1 Duchesse de Nemours, White, late

7.8 E. G. Hill, Large, roses pink

8.3 Eugene Verdier, Pink

8.1 Evangeline, Pale pink

8.0 Festiva Maxima, White

8.5 Germaine Bigot, Cameo pink

8.2 Gismonda, Creamy white, late

7.8 Glorie de Touraine, Velvety red, very late

7.0 Goliath, Dark pink

8.8 Grandiflora, Shell pink

8.8 Karl Rosenfield, Dark crimson

8.6 La Lorraine, Creamy white

8.1 Livingstone, Rose, late

7.6 Lord Kitchener, Brilliant red, early

7.3 Madam Bacquet, Velvety crimson, almost black

8.0 Marie Deroux, Flesh pink

Mary C. Lagergreen, Red

8.1 Mme. Calot, Flesh pink with buff

8.9 Mme. Emile Lemoine, Flesh white

7.7 Mons. Krelage, Deep red

Othello, Dark red

8.6 Primevere, White with yellow center

8.7 Reine Hortensse, Soft pink

9.0 Sarah Bernhardt, Late pink

8.1 Stanley, Pink with lilac

8.1 Susette, Rose pink

7.1 Umbeliata Rosea, Pink and cream

7.9 Victor Hugo, Crimson red (similar to Felix Crousse)
Price each in lots of

10

$\$ .40$

.75

.75

.20

.20

.20

1.00

.40

.75

.20

.30

.50

1.00

.40

.40

.75

.75

.40

.60

.40

.50

.75

.20

.60

.50

.75

.75

.60

.75

.40

.30

.20

.40
100

$\$ .35$

.60

.18

.18

.18

.35

.18

.25

.40

.75

.35

.35

.60

.35

.50

.35

.18

.50

.40 



\section{8 ? \\ J. F. MUNSELL \\ GROWER OF PEONIESANDGLADIOLI \\ ASHTABULA, OHIO

I have a small supply of the newer Peonies which I offer for Fall Delivery in 3 eye roots as follows:

Cash with order. 5 per cent discount from below if order amounts to $\$ 25.00$ or more.

Rating

8.0 Aviator Raymond, Cherry red

8.4 Chestine Gowdy, Shell pink

8.7 Claire Dubois, Pink

7.8 E. G. Hill, Carmine rose

8.3 Eugene Verdier, Pink

8.4 Felix Crousse, Red

9.3 Festiva Maxima, White

9.1 Frances Willard, White

8.9 Georgiana Shaylor, Rose pink

8.2 Gismonda, Creamy white

7.6 Gloire de Touraine, Red

8.8 Grandiflora (R), Shell pink

8.8 Karl Rosenfield, Red

7.8 La Fayette, Pink

9.0 La France, Appleblossom pink

8.1 Livingstone, Pink

8.4 Lora Dexheimer, Crimson

7.6 Lord Kitchener, Red

8.9 M. Jacquin, White, water lily

8.9 Marie Crousse, Shell pink

8.0 Marie Deroux, Flesh pink

9.0 Milton Hill, Flesh pink, late

8.6 Mme. Auguste Dessert, Soft pink

7.9 Mme. Ducel, Rose

8.9 Mme. Emile Lemoine, White

8.8 Mme. Gaudichau, Crimson

8.5 Mne. Manchet, Pink

9.2 Mons. Jules Elie, Pink

7.7 Mons. Krelage, Red, late

8.6 Officinalis Rubra Plena, Red

Officinalis Mut. Alba, White

8.7 Reine Hortense (President Taft), Pink

7.9 Ruth Brand, Pink

9.0 Sarah Bernhardt, Light pink

9.1 Souv. de Louis Bigot, Salmon pink

9.8 Therese, Violet rose

8.3 Venus, Light pink

8.2 Victor de la Marne, Crimson

7.9 Victor Hugo, Red

9.3 Walter Faxon, Rose
Price each in lots of

One
2.00
1.00
1.00

1.00

.50

.50

2.00

3.00

1.00

1.00

1.00

1.00

1.00

4.00

.75

2.00

.75

.75

1.00

1.00

1.00

1.50

.50

.75

3.00

3.00

.75

.75

.50

.50

.75

1.50

1.00

4.00

3.00

.75

2.00

.50

3.00 $\underset{\$ .75}{\text { Five }} \$ 25$

.40

.40

.30

.75

.65

.60

.50

.40

.50

.50

.50

.40

.35

.30

.50

.65

.60

.50

.40 
